\title{
Generalized Sampling Expansions Associated with Quaternion Fourier Transform
}

\author{
Dong Cheng* and Kit Ian Kou ${ }^{\dagger}$ \\ Department of Mathematics, Faculty of Science and Technology, University of Macau, \\ Macao, China
}

\begin{abstract}
Quaternion-valued signals along with quaternion Fourier transforms (QFT) provide an effective framework for vector-valued signal and image processing. However, the sampling theory of quaternion valued signals has not been well developed. In this paper, we present the generalized sampling expansions associated with QFT by using the generalized translation and convolution. We show that a $\sigma$-bandlimited quaternion valued signal in QFT sense can be reconstructed from the samples of output signals of $M$ linear systems based on QFT. Quaternion linear canonical transform (QLCT) is a generalization of QFT with six parameters. Using the relationship between QFT, we derive the sampling formula for $\sigma$-bandlimited quaternion-valued signal in QLCT sense. Examples are given to illustrate our results.
\end{abstract}

Keywords: Quaternion Fourier transform; quaternion linear canonical transform; sampling expansions; generalized translation; convolution theorem

\section{Introduction}

Generalized sampling expansions (GSE) developed by Papoulis [1] indicates that a $\sigma$ bandlimited signal can be reconstructed from the samples of output signals of $M$ linear systems. Namely,

$$
f(t)=\sum_{n=-\infty}^{\infty} \sum_{m=1}^{M} g_{m}(n T) y_{m}(t-n T)
$$

*chengdong720@163.com

†Corresponding author: kikou@umac.mo 
where $g_{m}(1 \leq m \leq M)$ are output signals of $M$ linear systems, $y_{m}(1 \leq m \leq M)$ are determined by a linear simultaneous equations whose coefficients are generated by system functions. Some classical sampling expansions, for instance, Shannon sampling expansions are special cases of Papoulis' result by choosing specific systems.

Over the years, the GSE has been extended in different ways. Hoskins and Pinto [2] extended the GSE to bandlimited distribution functions. A multidimensional extension of GSE was introduced by Cheung [3] for real-valued functions. While, Wei, Ran and Li [4] presented the GSE with generalized integral transformation, such as fractional Fourier transform. In this paper, higher-dimensional extension of GSE to quaternion-valued functions are studied. By powerful modelling of rotation and orientation, quaternion have shown advantages in physical and engineering applications such as computer graphics [5, 6] and robotics [7]. Furthermore, QFT has been regarded as a useful analysis tool in color image and signal processing $[8,9,10,11,12]$ in recently years. Therefore, it is desirable to define a system based on QFT to analyze quaternion-valued signals. Moreover, it is worthwhile and interesting to investigate the GSE using the samples of output signals of $M$ linear systems based on QFT. However, for the non-commutativity of the quaternion multiplication, the desirable shift property of classical Fourier transform is no longer available for QFT. Meanwhile, an crucial tool in signal processing called convolution theorem does not hold for QFT as well. The purpose of this paper is to overcome these problems and investigate the GSE. In this paper, we propose a novel translation of quaternion-valued signals and apply it to deduce the convolution theorem of QFT. More importantly, we present the GSE associated with QFT by proposed translation and convolution.

The rest of the paper is organized as follows. In the next section, we review QFT and some of its properties such as Plancherel theorem. Section 3 proposes a new translation and its corresponding convolution theorem. In Section 4, we present the generalized sampling expansion of bandlimited quaternion-valued signals in the sense of QFT. In Section 5, examples are presented to illustrate our results. In Section 6, we further discuss the sampling formula for $\sigma$-bandlimited quaternion valued signal in QLCT sense.

\section{Preliminary}

\subsection{Quaternion algebra}

Let's recall quaternion algebra $\mathbb{H}:=\left\{q=q_{0}+\mathbf{i} q_{1}+\mathbf{j} q_{2}+\mathbf{k} q_{3}: q_{0}, q_{1}, q_{2}, q_{3} \in \mathbb{R}\right\}$, where the imaginary elements $\mathbf{i}, \mathbf{j}$ and $\mathbf{k}$ obey $\mathbf{i}^{2}=\mathbf{j}^{2}=\mathbf{k}^{2}=\mathbf{i} \mathbf{j k}=-1$. For every quaternion $q=q_{0}+\underline{q}, \underline{q}=\mathbf{i} q_{1}+\mathbf{j} q_{2}+\mathbf{k} q_{3}$, the scalar and vector parts of $q$, are defined as $\operatorname{Sc}(q)=q_{0}$ and $\operatorname{Vec}(q)=q$, respectively. If $q=\operatorname{Vec}(q)$, then $q$ is called pure imaginary quaternion. The quaternion conjugate is defined by $\bar{q}=q_{0}-\underline{q}=q_{0}-\mathbf{i} q_{1}-\mathbf{j} q_{2}-\mathbf{k} q_{3}$, and the norm $|q|$ of $q$ defined as $|q|^{2}=q \bar{q}=\bar{q} q=\sum_{m=0}^{m=3} q_{m}^{2}$. Then we have

$$
\overline{\bar{q}}=q, \quad \overline{p+q}=\bar{p}+\bar{q}, \quad \overline{p q}=\bar{q} \bar{p}, \quad|p q|=|p||q|, \quad \forall p, q \in \mathbb{H} .
$$


Using the conjugate and norm of $q$, one can define the inverse of $q \in \mathbb{H} \backslash\{0\}$ as $q^{-1}=\bar{q} /|q|^{2}$.

The quaternion exponential function $\mathbf{e}^{q}$ is defined by means of an infinite series as $\mathbf{e}^{q}:=$ $\sum_{n=0}^{\infty} \frac{q^{n}}{n !}$. Analogous to the complex case one may derive a closed-form representation: $\mathbf{e}^{q}=\mathbf{e}^{q_{0}}\left(\cos |\underline{q}|+\frac{q}{|\underline{q}|} \sin |\underline{q}|\right)$.

Let $\mathbf{X}$ be a Lebesgue measurable subset of $\mathbb{R}^{2}$, the left $\mathbb{H}$-module $L^{p}(\mathbf{X}, \mathbb{H})(p=1,2)$ consists of all $\mathbb{H}$-valued functions whose $p$ th power is Lebesgue integrable on $\mathbf{X}$. The left quaternionic inner product of $f, g \in L^{2}(\mathbf{X}, \mathbb{H})$ is defined by

$$
\langle f, g\rangle:=\int_{\mathbf{X}} f\left(x_{1}, x_{2}\right) \overline{g\left(x_{1}, x_{2}\right)} d x_{1} d x_{2} .
$$

In fact, $L^{2}(\mathbf{X}, \mathbb{H})$ is a left quaternionic Hilbert space with inner product $\langle\cdot, \cdot\rangle$ (see [13]). Therefore, if $\left\{e_{n}\right\}$ is an orthonormal basis of $L^{2}(\mathbf{X}, \mathbb{H})$, then

$$
\langle f, g\rangle=\sum_{n}\left\langle f, e_{n}\right\rangle\left\langle e_{n}, g\right\rangle
$$

holds for all $f, g \in L^{2}(\mathbf{X}, \mathbb{H})$. This is a desirable property of quaternionic Hilbert space [14].

\subsection{Quaternion Fourier transform}

The quaternion Fourier transform (QFT) was first introduced by Ell to analyze partial differential equations [15]. Since then, QFT were applied to color image processing effectively $[8,9,10,12]$. There are different types of QFT [16] due to the non-commutativity of the quaternion multiplication . In [17], the authors investigated the properties of distinct types of QFT thoroughly, especially the following right-sided QFT.

Definition 2.1 (QFT) For every $f \in L^{1}\left(\mathbb{R}^{2}, \mathbb{H}\right)$, the right-sided QFT of $f$ is defined by

$$
(\mathcal{F} f)\left(\omega_{1}, \omega_{2}\right):=\frac{1}{2 \pi} \int_{\mathbb{R}^{2}} f\left(x_{1}, x_{2}\right) \mathbf{e}^{-\mathbf{i} \omega_{1} x_{1}} \mathbf{e}^{-\mathbf{j} \omega_{2} x_{2}} d x_{1} d x_{2} .
$$

If $\mathcal{F} f$ is also in $L^{1}\left(\mathbb{R}^{2}, \mathbb{H}\right)$, the inversion QFT formula (see $\left.[18,17]\right)$ holds, that is

$$
f\left(x_{1}, x_{2}\right)=\left(\mathcal{F}^{-1} \mathcal{F} f\right)\left(x_{1}, x_{2}\right):=\frac{1}{2 \pi} \int_{\mathbb{R}^{2}}(\mathcal{F} f)\left(\omega_{1}, \omega_{2}\right) \mathbf{e}^{\mathrm{j} \omega_{2} x_{2}} \mathbf{e}^{\mathrm{i} \omega_{1} x_{1}} d \omega_{1} \omega_{2},
$$

for almost every $\left(x_{1}, x_{2}\right) \in \mathbb{R}^{2}$. By Plancherel theorem (see $[18,17]$ ), the QFT can be extended to $L^{2}\left(\mathbb{R}^{2}, \mathbb{H}\right)$. As an operator on $L^{2}\left(\mathbb{R}^{2}, \mathbb{H}\right)$, the QFT $\Psi$ is a bijection and the Parseval's identity $\|\Psi f\|_{2}=\|f\|_{2}$ holds.

Remark 2.2 Since $\Psi\left(\Psi^{-1}\right)$ coincides with $\mathcal{F}\left(\mathcal{F}^{-1}\right)$ in $L^{1}\left(\mathbb{R}^{2}, \mathbb{H}\right) \cap L^{2}\left(\mathbb{R}^{2}, \mathbb{H}\right)$. For simplicity of notations, in the following, by capital letter $F$, we mean the QFT of $f \in$ $L^{1}\left(\mathbb{R}^{2}, \mathbb{H}\right) \cup L^{2}\left(\mathbb{R}^{2}, \mathbb{H}\right)$ if no otherwise specified. 


\section{Generalized translation and convolution}

A generalized translation related to the general integral transform with kernel $K(\omega, t)$ was introduced in [19]. Motivated by this study, we define the generalized translation to the quaternion-valued signals.

Definition 3.1 Let $f \in L^{1}\left(\mathbb{R}^{2}, \mathbb{H}\right) \cup L^{2}\left(\mathbb{R}^{2}, \mathbb{H}\right)$ and $F \in L^{1}\left(\mathbb{R}^{2}, \mathbb{H}\right)$. The generalized translation related to QFT is defined by

$$
f\left(x_{1} \ominus y_{1}, x_{2} \ominus y_{2}\right):=\frac{1}{2 \pi} \int_{\mathbb{R}^{2}} \mathbf{e}^{-\mathbf{i} \omega_{1} y_{1}} \mathbf{e}^{-\mathbf{j} \omega_{2} y_{2}} F\left(\omega_{1}, \omega_{2}\right) \mathbf{e}^{\mathbf{j} \omega_{2} x_{2}} \mathbf{e}^{\mathbf{i} \omega_{1} x_{1}} d \omega_{1} d \omega_{2} .
$$

Remark 3.2 In the complex case, the 2D Fourier transform of $f\left(x_{1}-y_{1}, x_{2}-y_{2}\right)$ with respect to $\left(x_{1}, x_{2}\right)$ is $\mathbf{e}^{-\mathbf{i} \omega_{1} y_{1}} \mathbf{e}^{-\mathbf{i} \omega_{2} y_{2}} \widehat{f}\left(\omega_{1}, \omega_{2}\right)$, where $\widehat{f}\left(\omega_{1}, \omega_{2}\right)$ is the $2 D$ Fourier transform of complex-valued function $f$. Therefore, the generalized translation $f\left(x_{1} \ominus y_{1}, x_{2} \ominus y_{2}\right)$, in some sense, is an analogue of $f\left(x_{1}-y_{1}, x_{2}-y_{2}\right)$. Moreover, $f\left(x_{1} \ominus y_{1}, x_{2} \ominus y_{2}\right)$ coincides with $f\left(x_{1}-y_{1}, x_{2}-y_{2}\right)$ for some special $f\left(x_{1}, x_{2}\right)$ (see Example 5.1).

Suppose that $h \in L^{1}\left(\mathbb{R}^{2}, \mathbb{H}\right) \cup L^{2}\left(\mathbb{R}^{2}, \mathbb{H}\right), H \in L^{1}\left(\mathbb{R}^{2}, \mathbb{H}\right)$ and $f \in L^{1}\left(\mathbb{R}^{2}, \mathbb{H}\right)$, then

$$
\int_{\mathbb{R}^{4}}\left|f\left(y_{1}, y_{2}\right) H\left(\omega_{1}, \omega_{2}\right)\right| d y_{1} d y_{2} d \omega_{1} d \omega_{2}<\infty
$$

Therefore, by Fubini's Theorem, we have

$$
\begin{aligned}
& \frac{1}{2 \pi} \int_{\mathbb{R}^{2}} f\left(y_{1}, y_{2}\right)\left(x_{1} \ominus y_{1}, x_{2} \ominus y_{2}\right) d y_{1} d y_{2} \\
= & \frac{1}{4 \pi^{2}} \int_{\mathbb{R}^{2}} d y_{1} d y_{2} f\left(y_{1}, y_{2}\right) \int_{\mathbb{R}^{2}} \mathbf{e}^{-\mathbf{i} \omega_{1} y_{1}} \mathbf{e}^{-\mathbf{j} \omega_{2} y_{2}} H\left(\omega_{1}, \omega_{2}\right) \mathbf{e}^{\mathbf{j} \omega_{2} x_{2}} \mathbf{e}^{\mathbf{i} \omega_{1} x_{1}} d \omega_{1} d \omega_{2} \\
= & \frac{1}{2 \pi} \int_{\mathbb{R}^{2}} F\left(\omega_{1}, \omega_{2}\right) H\left(\omega_{1}, \omega_{2}\right) \mathbf{e}^{\mathbf{j} \omega_{2} x_{2}} \mathbf{e}^{\mathbf{i} \omega_{1} x_{1}} d \omega_{1} d \omega_{2} \\
= & \left(\mathcal{F}^{-1} G\right)\left(x_{1}, x_{2}\right)
\end{aligned}
$$

where $G=F H$. Thus it is reasonable to define the following generalized convolution.

Definition 3.3 Let $f, h, G=F H \in L^{1}\left(\mathbb{R}^{2}, \mathbb{H}\right) \cup L^{2}\left(\mathbb{R}^{2}, \mathbb{H}\right)$. The convolution of $f$ and $h$ is defined by

$$
(f \star h)\left(x_{1}, x_{2}\right):=\left(\mathcal{F}^{-1} G\right)\left(x_{1}, x_{2}\right) .
$$

Obviously, The QFT of $f \star h$ is $F H$.

Theorem 3.4 If any of the following conditions is satisfied.

1. If $h \in L^{1}\left(\mathbb{R}^{2}, \mathbb{H}\right) \cup L^{2}\left(\mathbb{R}^{2}, \mathbb{H}\right), H \in L^{1}\left(\mathbb{R}^{2}, \mathbb{H}\right)$ and $f \in L^{1}\left(\mathbb{R}^{2}, \mathbb{H}\right)$.

2. If $h \in L^{1}\left(\mathbb{R}^{2}, \mathbb{H}\right) \cup L^{2}\left(\mathbb{R}^{2}, \mathbb{H}\right)$, $H \in L^{1}\left(\mathbb{R}^{2}, \mathbb{H}\right) \cap L^{2}\left(\mathbb{R}^{2}, \mathbb{H}\right)$ and $f \in L^{2}\left(\mathbb{R}^{2}, \mathbb{H}\right)$. 
Then

$$
(f \star h)\left(x_{1}, x_{2}\right)=\frac{1}{2 \pi} \int_{\mathbb{R}^{2}} f\left(y_{1}, y_{2}\right)\left(x_{1} \ominus y_{1}, x_{2} \ominus y_{2}\right) d y_{1} d y_{2} .
$$

Proof. The first case is obviously true. We now prove the second case. Since $f \in$ $L^{2}\left(\mathbb{R}^{2}, \mathbb{H}\right)$, then $F \in L^{2}\left(\mathbb{R}^{2}, \mathbb{H}\right)$ by Plancherel theorem. Moreover, there is a sequence $\left\{f_{n}\right\}$ in $L^{1}\left(\mathbb{R}^{2}, \mathbb{H}\right) \cap L^{2}\left(\mathbb{R}^{2}, \mathbb{H}\right)$ converging to $f$ in $L^{2}$ norm such that $F=\lim _{n \rightarrow \infty} . F_{n}=\lim _{n \rightarrow \infty} \mathcal{F} f_{n}$. Note that $H \in L^{2}\left(\mathbb{R}^{2}, \mathbb{H}\right)$. Therefore, by Hölder inequality, $F H \in L^{1}\left(\mathbb{R}^{2}, \mathbb{H}\right)$ and

$$
\begin{aligned}
& (f \star h)\left(x_{1}, x_{2}\right) \\
= & \frac{1}{2 \pi} \int_{\mathbb{R}^{2}} F\left(\omega_{1}, \omega_{2}\right) H\left(\omega_{1}, \omega_{2}\right) \mathbf{e}^{\mathrm{j} \omega_{2} x_{2}} \mathbf{e}^{\mathbf{i} \omega_{1} x_{1}} d \omega_{1} d \omega_{2} \\
= & \lim _{n \rightarrow \infty} \frac{1}{2 \pi} \int_{\mathbb{R}^{2}} F_{n}\left(\omega_{1}, \omega_{2}\right) H\left(\omega_{1}, \omega_{2}\right) \mathbf{e}^{\mathbf{j} \omega_{2} x_{2}} \mathbf{e}^{\mathbf{i} \omega_{1} x_{1}} d \omega_{1} d \omega_{2} \\
= & \lim _{n \rightarrow \infty} \frac{1}{4 \pi^{2}} \int_{\mathbb{R}^{4}} d y_{1} d y_{2} f_{n}\left(y_{1}, y_{2}\right) \mathbf{e}^{-\mathbf{i} \omega_{1} y_{1}} \mathbf{e}^{-\mathbf{j} \omega_{2} y_{2}} H\left(\omega_{1}, \omega_{2}\right) \mathbf{e}^{\mathbf{j} \omega_{2} x_{2}} \mathbf{e}^{\mathbf{i} \omega_{1} x_{1}} d \omega_{1} d \omega_{2} \\
= & \lim _{n \rightarrow \infty} \frac{1}{2 \pi} \int_{\mathbb{R}^{2}} f_{n}\left(y_{1}, y_{2}\right)\left(x_{1} \ominus y_{1}, x_{2} \ominus y_{2}\right) d y_{1} d y_{2} \\
= & \frac{1}{2 \pi} \int_{\mathbb{R}^{2}} f\left(y_{1}, y_{2}\right)\left(x_{1} \ominus y_{1}, x_{2} \ominus y_{2}\right) d y_{1} d y_{2} .
\end{aligned}
$$

The interchange of integral and limit is permissible for the continuity of inner product.

\section{The GSE associated with QFT}

We give a definition of bandlimited signals in QFT sense.

Definition 4.1 (bandlimited) A signal $f\left(x_{1}, x_{2}\right)$ is $\sigma$-bandlimited in QFT sense if it can be expressed as

$$
f\left(x_{1}, x_{2}\right)=\frac{1}{2 \pi} \int_{I} F\left(\omega_{1}, \omega_{2}\right) \mathbf{e}^{\mathbf{j} \omega_{2} x_{2}} \mathbf{e}^{\mathbf{i} \omega_{1} x_{1}} d \omega_{1} d \omega_{2}
$$

where $F \in L^{2}(I, \mathbb{H})$ and $I=[-\sigma, \sigma]^{2}$. For any $\sigma>0$, denote by $\mathbf{B}_{\sigma}^{q}$ the totality of the $\sigma$-bandlimited signals in QFT sense.

If $f \in \mathbf{B}_{\sigma}^{q}$, by Plancherel theorem, we have $f \in L^{2}\left(\mathbb{R}^{2}, \mathbb{H}\right)$ and the QFT of $f$ is $F$. In this part, we show that $f$ can be reconstructed from the samples of the inverse QFT of $M:=m^{2}$ functions $G_{k}=F H_{k},(k=1,2, \ldots, M)$ if $H_{k}$ satisfy suitable conditions.

Let $T:=\frac{m \pi}{\sigma}, c:=\frac{2 \sigma}{m}=\frac{2 \pi}{T}$ and

$$
I_{n_{1} n_{2}}:=\left[-\sigma+\left(n_{1}-1\right) c,-\sigma+n_{1} c\right] \times\left[-\sigma+\left(n_{2}-1\right) c,-\sigma+n_{2} c\right] .
$$


Then

$$
f\left(x_{1}, x_{2}\right)=\sum_{n_{1}=0}^{m-1} \sum_{n_{2}=0}^{m-1} \frac{1}{2 \pi} \int_{I_{11}} F\left(\omega_{1}+n_{1} c, \omega_{2}+n_{2} c\right) \mathbf{e}^{\mathbf{j}\left(\omega_{2}+n_{2} c\right) x_{2}} \mathbf{e}^{\mathbf{i}\left(\omega_{1}+n_{1} c\right) x_{1}} d \omega_{1} d \omega_{2} .
$$

To state our results, we need some further notations. Let

$$
\left\{\begin{array}{l}
a_{n_{1} n_{2}}\left(\omega_{1}, \omega_{2}\right):=F\left(\omega_{1}+\left(n_{1}-1\right) c, \omega_{2}+\left(n_{2}-1\right) c\right) \\
b_{n_{1} n_{2}}\left(\omega_{1}, \omega_{2}, x_{1}, x_{2}\right):=\mathbf{e}^{\mathbf{j}\left(\omega_{2}+\left(n_{2}-1\right) c\right) x_{2}} \mathbf{e}^{\mathbf{i}\left(\omega_{1}+\left(n_{1}-1\right) c\right) x_{1}} \\
r_{n_{1} n_{2}}^{k}\left(\omega_{1}, \omega_{2}\right):=H_{k}\left(\omega_{1}+\left(n_{1}-1\right) c, \omega_{2}+\left(n_{2}-1\right) c\right)
\end{array}\right.
$$

They form the following vectors or matrices:

$$
\left\{\begin{array}{l}
\vec{F}\left(\omega_{1}, \omega_{2}\right):=(A(1,:), A(2,:), \ldots, A(m,:))^{T}, \\
\vec{E}\left(\omega_{1}, \omega_{2}, x_{1}, x_{2}\right):=(B(1,:), B(2,:), \ldots, B(m,:))^{T}, \\
\overrightarrow{H_{k}}\left(\omega_{1}, \omega_{2}\right):=\left(R_{k}(1,:), R_{k}(2,:), \ldots, R_{k}(m,:)\right)^{T}, \\
\underline{H}\left(\omega_{1}, \omega_{2}\right):=\left(\underline{H}_{1}\left(\omega_{1}, \omega_{2}\right), \vec{H}_{2}\left(\omega_{1}, \omega_{2}\right), \ldots, \underline{H}_{M}\left(\omega_{1}, \omega_{2}\right)\right), \\
\vec{G}\left(\omega_{1}, \omega_{2}\right):=\left(\widetilde{G}_{1}\left(\omega_{1}, \omega_{2}\right), \widetilde{G}_{2}\left(\omega_{1}, \omega_{2}\right), \ldots, \widetilde{G}_{M}\left(\omega_{1}, \omega_{2}\right)\right)=\vec{F}\left(\omega_{1}, \omega_{2}\right)^{T} \underline{H}\left(\omega_{1}, \omega_{2}\right),
\end{array}\right.
$$

where $A, B, R_{k}$ are $m \times m$ matrices with entries $a_{n_{1} n_{2}}\left(\omega_{1}, \omega_{2}\right), b_{n_{1} n_{2}}\left(\omega_{1}, \omega_{2}, x_{1}, x_{2}\right)$, $r_{n_{1} n_{2}}^{k}\left(\omega_{1}, \omega_{2}\right)$ respectively. Assume that $\underline{H}$ is invertible for every $\left(\omega_{1}, \omega_{2}\right) \in I_{11}$. Denote the inverse of $\underline{H}$ by

$$
\underline{H}^{-1}\left(\omega_{1}, \omega_{2}\right):=\left(\underline{Q}_{1}\left(\omega_{1}, \omega_{2}\right) ; \underline{Q}_{2}\left(\omega_{1}, \omega_{2}\right) ; \ldots ; \overrightarrow{Q_{M}}\left(\omega_{1}, \omega_{2}\right)\right)
$$

where $\underline{Q_{k}}\left(\omega_{1}, \omega_{2}\right)=\left(Q_{k}(1,:), Q_{k}(2,:), \ldots, Q_{k}(m,:)\right)$ and $Q_{k}=\left(q_{n_{1} n_{2}}^{k}\left(\omega_{1}, \omega_{2}\right)\right)_{m \times m}$. Then (4.2) becomes

$$
f\left(x_{1}, x_{2}\right)=\frac{1}{2 \pi} \int_{I_{11}} \vec{F}\left(\omega_{1}, \omega_{2}\right) \vec{E}\left(\omega_{1}, \omega_{2}, x_{1}, x_{2}\right) d \omega_{1}, \omega_{2}
$$

In the complex case, $\vec{E}$ only depends on $\left(x_{1}, x_{2}\right)$ (see [3]). However, due to the noncommutativity of the quaternion algebra, $\vec{E}$ depends on both $\left(x_{1}, x_{2}\right)$ and $\left(\omega_{1}, \omega_{2}\right)$ in quaternionic case.

Lemma 4.2 If $H_{k} \in L^{2}(I, \mathbb{H})$, then samples of the inverse $Q F T$ of $G_{k}(k=1,2, \ldots, M)$ can be expressed as:

$$
g_{k}\left(n_{1} T, n_{2} T\right)=\frac{1}{2 \pi} \int_{I_{11}} \widetilde{G}_{k}\left(\omega_{1}, \omega_{2}\right) \mathbf{e}^{\mathrm{j} \omega_{2} n_{2} T} \mathbf{e}^{\mathrm{i} \omega_{1} n_{1} T} d \omega_{1} d \omega_{2}
$$

where $I_{11}$ is given in (4.1). 
Proof. Since $H_{k} \in L^{2}(I, \mathbb{H})$, then $G_{k} \in L^{2}(I, \mathbb{H})$,

$$
\begin{aligned}
g_{k}\left(x_{1}, x_{2}\right) & =\frac{1}{2 \pi} \int_{I} G_{k}\left(\omega_{1}, \omega_{2}\right) \mathbf{e}^{\mathbf{j} \omega_{2} x_{2}} \mathbf{e}^{\mathbf{i} \omega_{1} x_{1}} d \omega_{1} d \omega_{2} \\
& =\frac{1}{2 \pi} \int_{I} F\left(\omega_{1}, \omega_{2}\right) H_{k}\left(\omega_{1}, \omega_{2}\right) \mathbf{e}^{\mathbf{j} \omega_{2} x_{2}} \mathbf{e}^{\mathbf{i} \omega_{1} x_{1}} d \omega_{1} d \omega_{2} \\
& =\sum_{l_{1}=1}^{m} \sum_{l_{2}=1}^{m} \frac{1}{2 \pi} \int_{I_{11}} a_{l_{1} l_{2}}\left(\omega_{1}, \omega_{2}\right) r_{l_{1} l_{2}}^{k}\left(\omega_{1}, \omega_{2}\right) b_{l_{1} l_{2}}\left(\omega_{1}, \omega_{2}, x_{1}, x_{2}\right) d \omega_{1} d \omega_{2} \\
& =\frac{1}{2 \pi} \int_{I_{11}} \sum_{l_{1}=1}^{m} \sum_{l_{2}=1}^{m} a_{l_{1} l_{2}}\left(\omega_{1}, \omega_{2}\right) r_{l_{1} l_{2}}^{k}\left(\omega_{1}, \omega_{2}\right) b_{l_{1} l_{2}}\left(\omega_{1}, \omega_{2}, x_{1}, x_{2}\right) d \omega_{1} d \omega_{2}
\end{aligned}
$$

and $b_{l_{1} l_{2}}\left(\omega_{1}, \omega_{2}, n_{1} T, n_{2} T\right)=\mathbf{e}^{\mathbf{j} \omega_{2} n_{2} T} \mathbf{e}^{\mathbf{i} \omega_{1} n_{1} T}$ which is independent of $l_{1} l_{2}$. Therefore

$$
\begin{aligned}
& \sum_{l_{1}=1}^{m} \sum_{l_{2}=1}^{m} a_{l_{1} l_{2}}\left(\omega_{1}, \omega_{2}\right) r_{l_{1} l_{2}}^{k}\left(\omega_{1}, \omega_{2}\right) b_{l_{1} l_{2}}\left(\omega_{1}, \omega_{2}, x_{1}, x_{2}\right) \\
= & \vec{F}\left(\omega_{1}, \omega_{2}\right)^{T} \underline{\hat{H}_{k}}\left(\omega_{1}, \omega_{2}\right) \mathbf{e}^{\mathbf{j} \omega_{2} n_{2} T} \mathbf{e}^{\mathbf{i} \omega_{1} n_{1} T} .
\end{aligned}
$$

It follows that

$$
\begin{aligned}
g_{k}\left(n_{1} T, n_{2} T\right) & =\frac{1}{2 \pi} \int_{I_{11}} \vec{F}\left(\omega_{1}, \omega_{2}\right)^{T} \underline{\vec{H}}_{k}\left(\omega_{1}, \omega_{2}\right) \mathbf{e}^{\mathrm{j} \omega_{2} n_{2} T} \mathbf{e}^{\mathrm{i} \omega_{1} n_{1} T} d \omega_{1}, \omega_{2} \\
& =\frac{1}{2 \pi} \int_{I_{11}} \widetilde{G}_{k}\left(\omega_{1}, \omega_{2}\right) \mathbf{e}^{\mathrm{j} \omega_{2} n_{2} T} \mathbf{e}^{\mathbf{i} \omega_{1} n_{1} T} d \omega_{1} \omega_{2} .
\end{aligned}
$$

Lemma 4.3 Suppose that $q_{l_{1} l_{2}}^{k} \in L^{2}\left(I_{11}, \mathbb{H}\right)$ and let

$$
\widetilde{q}_{n_{1} n_{2}}^{k}\left(\omega_{1}, \omega_{2}\right)=q_{n_{1} n_{2}}^{k}\left(\omega_{1}-\left(n_{1}-1\right) c, \omega_{2}-\left(n_{2}-1\right) c\right) \chi_{I_{n_{1} n_{2}}}\left(\omega_{1}, \omega_{2}\right)
$$

and

$$
Y_{k}\left(\omega_{1}, \omega_{2}\right)=\frac{T^{2}}{2 \pi} \sum_{n_{1}=1}^{m} \sum_{n_{2}=1}^{m} \widetilde{q}_{n_{1} n_{2}}^{k}\left(\omega_{1}, \omega_{2}\right) .
$$

Then for every $\left(n_{1}, n_{2}\right) \in \mathbb{Z}^{2}, \frac{4 \pi^{2}}{T^{2}} y_{k}\left(x_{1} \ominus n_{1} T, x_{2} \ominus n_{2} T\right)$ equals to

$$
\int_{I_{11}} \mathbf{e}^{-\mathbf{i} \omega_{1} n_{1} T} \mathbf{e}^{-\mathbf{j} \omega_{2} n_{2} T} \sum_{l_{1}=1}^{m} \sum_{l_{2}=1}^{m} q_{l_{1} l_{2}}^{k}\left(\omega_{1}, \omega_{2}\right) b_{l_{1} l_{2}}\left(\omega_{1}, \omega_{2}, x_{1}, x_{2}\right) d \omega_{1} \omega_{2}
$$

where $y_{k}$ is the inverse QFT of $Y_{k}$.

Proof. By rewriting $y_{k}\left(x_{1} \ominus n_{1} T, x_{2} \ominus n_{2} T\right)$ in the form of (3.1) and substituting $\mathbf{e}^{\mathbf{j} \omega_{2} x_{2}} \mathbf{e}^{\mathbf{i} \omega_{1} x_{1}}$ and $\mathbf{e}^{-\mathbf{i} \omega_{1} n_{1} T} \mathbf{e}^{-\mathbf{j} \omega_{2} n_{2} T}$ with $b_{l_{1} l_{2}}\left(\omega_{1}-\left(l_{1}-1\right) c, \omega_{2}-\left(l_{2}-1\right) c, x_{1}, x_{2}\right)$ and

$$
\overline{b_{n_{1} n_{2}}\left(\omega_{1}-\left(l_{1}-1\right) c, \omega_{2}-\left(l_{2}-1\right) c, n_{1} T, n_{2} T\right)}
$$




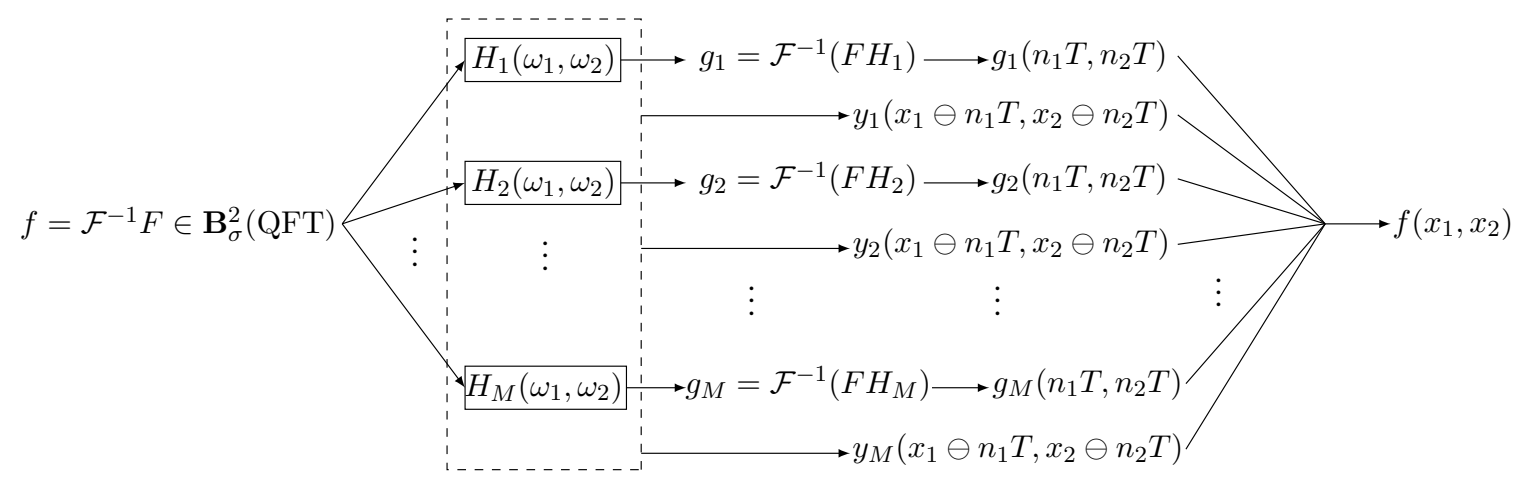

Figure 1: Diagram of GSE associated with QFT.

respectively, we obtain

$$
\begin{aligned}
& \frac{2 \pi}{T^{2}} \int_{\mathbb{R}^{2}} \mathbf{e}^{-\mathbf{i} \omega_{1} n_{1} T} \mathbf{e}^{-\mathbf{j} \omega_{2} n_{2} T} Y_{k}\left(\omega_{1}, \omega_{2}\right) \mathbf{e}^{\mathbf{j} \omega_{2} x_{2}} \mathbf{e}^{\mathbf{i} \omega_{1} x_{1}} d \omega_{1} d \omega_{2} \\
= & \sum_{l_{1}=1}^{m} \sum_{l_{2}=1}^{m} \int_{\mathbb{R}^{2}} \mathbf{e}^{-\mathbf{i} \omega_{1} n_{1} T} \mathbf{e}^{-\mathbf{j} \omega_{2} n_{2} T} \widetilde{q}_{l_{1} l_{2}}^{k}\left(\omega_{1}, \omega_{2}\right) \mathbf{e}^{\mathbf{j} \omega_{2} x_{2}} \mathbf{e}^{\mathbf{i} \omega_{1} x_{1}} d \omega_{1} d \omega_{2} \\
= & \sum_{l_{1}=1}^{m} \sum_{l_{2}=1}^{m} \int_{I_{l_{1} l_{2}}} \overline{b_{n_{1} n_{2}}\left(\omega_{1}-\left(l_{1}-1\right) c, \omega_{2}\left(l_{2}-1\right) c, n_{1} T, n_{2} T\right)} \\
& q_{l_{1} l_{2}}^{k}\left(\omega_{1}-\left(l_{1}-1\right) c, \omega_{2}-\left(l_{2}-1\right) c\right) b_{l_{1} l_{2}}\left(\omega_{1}-\left(l_{1}-1\right) c, \omega_{2}-\left(l_{2}-1\right) c, x_{1}, x_{2}\right) d \omega_{1} d \omega_{2} \\
= & \sum_{l_{1}=1}^{m} \sum_{l_{2}=1}^{m} \int_{I_{11}} \overline{b_{l_{1} l_{2}}\left(\omega_{1}, \omega_{2}, n_{1} T, n_{2} T\right)} q_{l_{1} l_{2}}^{k}\left(\omega_{1}, \omega_{2}\right) b_{l_{1} l_{2}}\left(\omega_{1}, \omega_{2}, x_{1}, x_{2}\right) d \omega_{1} d \omega_{2} \\
= & \int_{I_{11}} \mathbf{e}^{-\mathbf{i} \omega_{1} n_{1} T} \mathbf{e}^{-\mathbf{j} \omega_{2} n_{2} T} \sum_{l_{1}=1}^{m} \sum_{l_{2}=1}^{m} q_{l_{1} l_{2}}^{k}\left(\omega_{1}, \omega_{2}\right) b_{l_{1} l_{2}}\left(\omega_{1}, \omega_{2}, x_{1}, x_{2}\right) d \omega_{1} d \omega_{2} .
\end{aligned}
$$

which completes the proof.

Now we give the generalized sampling expansion associated with QFT.

Theorem 4.4 Let $H_{1}, H_{2}, \ldots, H_{M}$ such that
1. $H_{k} \in L^{2}(I, \mathbb{H})$,
2. $\underline{H}\left(\omega_{1}, \omega_{2}\right)$ is invertible for every $\left(\omega_{1}, \omega_{2}\right) \in I_{11}$ and $q_{l_{1} l_{2}}^{k} \in L^{2}\left(I_{11}, \mathbb{H}\right)$.

Then $f$ can be reconstructed from samples $g_{k}\left(n_{1} T, n_{2} T\right)$ of

$$
\begin{aligned}
g_{k}\left(x_{1}, x_{2}\right) & =\left(f \star h_{k}\right)\left(x_{1}, x_{2}\right) \\
& =\frac{1}{2 \pi} \int_{I} F\left(\omega_{1}, \omega_{2}\right) H_{k}\left(\omega_{1}, \omega_{2}\right) \mathbf{e}^{\mathrm{j} \omega_{2} x_{2}} \mathbf{e}^{\mathrm{i} \omega_{1} x_{1}} d \omega_{1} d \omega_{2} .
\end{aligned}
$$


More specifically,

$$
f\left(x_{1}, x_{2}\right)=\sum_{k=1}^{M} \sum_{n_{1}, n_{2}} g_{k}\left(n_{1} T, n_{2} T\right) y_{k}\left(x_{1} \ominus n_{1} T, x_{2} \ominus n_{2} T\right)
$$

where $y_{k}$ is the inverse QFT of $Y_{k}$.

Proof. Since $\underline{H}\left(\omega_{1}, \omega_{2}\right)$ is invertible for every $\left(\omega_{1}, \omega_{2}\right) \in I_{11}$ then

$$
\underline{H}^{-1}\left(\omega_{1}, \omega_{2}\right) \vec{E}\left(\omega_{1}, \omega_{2}, x_{1}, x_{2}\right)
$$

is a $m \times 1$ matrix and the $k$ th element equals to

$$
\sum_{l_{1}=1}^{m} \sum_{l_{2}=1}^{m} q_{l_{1} l_{2}}^{k}\left(\omega_{1}, \omega_{2}\right) b_{l_{1} l_{2}}\left(\omega_{1}, \omega_{2}, x_{1}, x_{2}\right)
$$

Therefore

$$
\begin{aligned}
f\left(x_{1}, x_{2}\right) & =\frac{1}{2 \pi} \int_{I_{11}} \vec{F}\left(\omega_{1}, \omega_{2}\right) \vec{E}\left(\omega_{1}, \omega_{2}, x_{1}, x_{2}\right) d \omega_{1} d \omega_{2} \\
& =\frac{1}{2 \pi} \int_{I_{11}} \vec{G}\left(\omega_{1}, \omega_{2}\right) \underline{H}\left(\omega_{1}, \omega_{2}\right)^{-1} \vec{E}\left(\omega_{1}, \omega_{2}, x_{1}, x_{2}\right) d \omega_{1} d \omega_{2} \\
& =\sum_{k=1}^{M} \frac{1}{2 \pi} \int_{I_{11}} \widetilde{G}_{k}\left(\omega_{1}, \omega_{2}\right) \sum_{l_{1}=1}^{m} \sum_{l_{2}=1}^{m} q_{l_{1} l_{2}}^{k}\left(\omega_{1}, \omega_{2}\right) b_{l_{1} l_{2}}\left(\omega_{1}, \omega_{2}, x_{1}, x_{2}\right) d \omega_{1} d \omega_{2}
\end{aligned}
$$

As $F, H_{k} \in L^{2}(I, \mathbb{H})$, it is easy to see that $\widetilde{G}_{k} \in L^{2}\left(I_{11}, \mathbb{H}\right)$. Also, if $q_{l_{1} l_{2}}^{k} \in L^{2}(I, \mathbb{H})$ then

$$
\sum_{l_{1}=1}^{m} \sum_{l_{2}=1}^{m} q_{l_{1} l_{2}}^{k}\left(\omega_{1}, \omega_{2}\right) b_{l_{1} l_{2}}\left(\omega_{1}, \omega_{2}, x_{1}, x_{2}\right) \in L^{2}\left(I_{11}, \mathbb{H}\right)
$$

for every $\left(x_{1}, x_{2}\right) \in \mathbb{R}^{2}$. Since $\left\{\frac{T}{2 \pi} \mathbf{e}^{-\mathbf{i} \omega_{1} n_{1} T} \mathbf{e}^{-\mathbf{j} \omega_{2} n_{2} T}\right\}_{\left(n_{1}, n_{2}\right) \in \mathbb{Z}^{2}}$ is an orthonormal basis of $L^{2}\left(I_{11}, \mathbb{H}\right)$. Therefore by invoking $(2.1)$ we have

$$
\begin{aligned}
& \frac{1}{2 \pi} \int_{I_{11}} \widetilde{G}_{k}\left(\omega_{1}, \omega_{2}\right) \sum_{l_{1}=1}^{m} \sum_{l_{2}=1}^{m} q_{l_{1} l_{2}}^{k}\left(\omega_{1}, \omega_{2}\right) b_{l_{1} l_{2}}\left(\omega_{1}, \omega_{2}, x_{1}, x_{2}\right) d \omega_{1} d \omega_{2} \\
= & \sum_{n_{1}, n_{2}} \frac{T^{2}}{8 \pi^{3}} \int_{I_{11}} \widetilde{G}_{k}\left(\omega_{1}, \omega_{2}\right) \mathbf{e}^{\mathbf{j} \omega_{2} n_{2} T} \mathbf{e}^{\mathbf{i} \omega_{1} n_{1} T} d \omega_{1} d \omega_{2} \\
& \int_{I_{11}} \mathbf{e}^{-\mathbf{i} \omega_{1} n_{1} T} \mathbf{e}^{-\mathbf{j} \omega_{2} n_{2} T} \sum_{l_{1}=1}^{m} \sum_{l_{2}=1}^{m} q_{l_{1} l_{2}}^{k}\left(\omega_{1}, \omega_{2}\right) b_{l_{1} l_{2}}\left(\omega_{1}, \omega_{2}, x_{1}, x_{2}\right) d \omega_{1} d \omega_{2}
\end{aligned}
$$

Hence, by Lemma 4.2 and Lemma 4.3, we obtain (4.5). 


\section{$5 \quad$ Examples}

Example 5.1 If $f$ is $\sigma$-bandlimited, we have

$$
f\left(x_{1}, x_{2}\right)=\sum_{n_{1}, n_{2}} f\left(n_{1} T, n_{2} T\right) \frac{\sin \left(\sigma x_{1}-n_{1} \pi\right) \sin \left(\sigma x_{2}-n_{2} \pi\right)}{\left(\sigma x_{1}-n_{1} \pi\right)\left(\sigma x_{2}-n_{2} \pi\right)}
$$

by choosing $m=1$ and $H_{1}\left(\omega_{1}, \omega_{2}\right)=1$, where $T=\frac{\pi}{\sigma}$. Let $\sigma^{\prime}=\rho \sigma$ with $\rho>1$ and $H\left(\omega_{1}, \omega_{2}\right)=H^{1}\left(\omega_{1}\right) H^{1}\left(\omega_{2}\right)$ with

$$
H^{1}\left(\omega_{1}\right)= \begin{cases}1, & \left|\omega_{1}\right| \leq \sigma \\ 0, & \left|\omega_{1}\right| \geq \sigma^{\prime}, \\ \frac{1}{(1-\rho) \sigma}\left|\omega_{1}\right|+\frac{\rho}{\rho-1}, & \sigma \leq\left|\omega_{1}\right| \leq \sigma^{\prime}\end{cases}
$$

Note that $f$ is $\sigma^{\prime}$-bandlimited. Therefore, by applying Theorem 4.4 with $M=1$ and $H\left(\omega_{1}, \omega_{2}\right)$ defined above, we have

$$
f\left(x_{1}, x_{2}\right)=\sum_{n_{1}, n_{2}} f\left(n_{1} T^{\prime}, n_{1} T^{\prime}\right) y\left(x_{1}-n_{1} T, x_{2}-n_{2} T\right)
$$

where

$$
y\left(x_{1}, x_{2}\right)=\frac{4\left(\sin ^{2} \frac{\rho \sigma x_{1}}{2}-\sin ^{2} \frac{\sigma x_{1}}{2}\right)\left(\sin ^{2} \frac{\rho \sigma x_{2}}{2}-\sin ^{2} \frac{\sigma x_{2}}{2}\right)}{x_{1}^{2} x_{2}^{2} \rho^{2}(\rho-1)^{2} \sigma^{4}}
$$

and $T^{\prime}=\frac{\pi}{\sigma^{\prime}}=\frac{T}{\rho}<T$. For any fixed $\left(x_{1}, x_{2}\right) \in \mathbb{R}^{2}$, (5.2) converges faster than (5.1). It illustrates that convergence rate of sampling series can be enhanced by increasing the sampling frequency.

Example 5.2 Express a $\sigma$-bandlimited function $f\left(x_{1}, x_{2}\right)$ from the samples $g\left(n_{1} T, n_{2} T\right)$ of the integral

$$
g\left(x_{1}, x_{2}\right)=\frac{1}{2 \pi} \int_{I} F\left(\omega_{1}, \omega_{2}\right) H\left(\omega_{1}, \omega_{2}\right) \mathbf{e}^{\mathbf{j} \omega_{2} x_{2}} \mathbf{e}^{\mathrm{i} \omega_{1} x_{1}} d \omega_{1}, \omega_{2}
$$

where $H\left(\omega_{1}, \omega_{2}\right)=\alpha \beta\left(\beta+\mathbf{j} \omega_{2}\right)^{-1}\left(\alpha+\mathbf{i} \omega_{1}\right)^{-1}(\alpha, \beta>0), I=[-\sigma, \sigma]^{2}, T=\frac{\pi}{\sigma}$. In fact, by Theorem 3.4, we have

$$
g\left(x_{1}, x_{2}\right)=\frac{1}{2 \pi} \int_{\mathbb{R}^{2}} f\left(y_{1}, y_{2}\right) \widetilde{h}\left(x_{1} \ominus y_{2}, x_{2} \ominus y_{2}\right) d y_{1} d y_{2}
$$

where

$$
\widetilde{h}\left(x_{1} \ominus y_{1}, x_{2} \ominus y_{2}\right)=\frac{1}{2 \pi} \int_{I} \mathbf{e}^{-\mathbf{i} \omega_{1} y_{1}} \mathbf{e}^{-\mathbf{j} \omega_{2} y_{2}} H\left(\omega_{1}, \omega_{2}\right) \mathbf{e}^{\mathbf{j} \omega_{2} x_{2}} \mathbf{e}^{\mathbf{i} \omega_{1} x_{1}} d \omega_{1} d \omega_{2} .
$$

It is easy to see that $H$ satisfies all conditions of Theorem 4.4. From (4.3), we have

$$
Y\left(\omega_{1}, \omega_{2}\right)=\frac{T^{2}\left(\alpha+\mathbf{i} \omega_{1}\right)\left(\beta+\mathbf{j} \omega_{2}\right)}{2 \pi \alpha \beta} \chi_{I}\left(\omega_{1}, \omega_{2}\right)
$$


By direct computation, $4 \pi T^{-2} y\left(x_{1} \ominus n_{1} T, x_{2} \ominus n_{2} T\right)$ is given by

$$
\begin{aligned}
& \frac{4 \sin \sigma\left(x_{1}-n_{1} T\right) \sin \sigma\left(x_{2}-n_{2} T\right)}{\left(x_{1}-n_{1} T\right)\left(x_{2}-n_{2} T\right)} \\
& -\frac{4 \sin \sigma\left(x_{2}+n_{2} T\right)\left[\sin \sigma\left(x_{1}-n_{1} T\right)-\sigma\left(x_{1}-n_{1} T\right) \cos \sigma\left(x_{1}-n_{1} T\right)\right]}{\alpha\left(x_{2}+n_{2} T\right)\left(x_{1}-n_{1} T\right)^{2}} \\
& -\frac{4 \sin \sigma\left(x_{1}-n_{1} T\right)\left[\sin \sigma\left(x_{2}-n_{2} T\right)-\sigma\left(x_{2}-n_{2} T\right) \cos \sigma\left(x_{2}-n_{2} T\right)\right]}{\beta\left(x_{1}-n_{1} T\right)\left(x_{2}-n_{2} T\right)^{2}} \\
& +\frac{4\left[\sin \sigma\left(x_{1}-n_{1} T\right)-\sigma\left(x_{1}-n_{1} T\right) \cos \sigma\left(x_{1}-n_{1} T\right)\right]}{\alpha \beta\left(x_{1}-n_{1} T\right)^{2}\left(x_{2}+n_{2} T\right)^{2}} \\
& \\
& \quad\left[\sin \sigma\left(x_{2}+n_{2} T\right)-\sigma\left(x_{2}+n_{2} T\right) \cos \sigma\left(x_{2}+n_{2} T\right)\right] .
\end{aligned}
$$

Example 5.3 Theorem 4.4 permits us to express a $\sigma$-bandlimited function $f\left(x_{1}, x_{2}\right)$ from its samples and samples of its partial derivatives. Let $m=2, T=\frac{2 \pi}{\sigma}, c=\sigma$ and $H_{1}\left(\omega_{1}, \omega_{2}\right)=1, H_{2}\left(\omega_{1}, \omega_{2}\right)=\mathbf{i} \omega_{1}, H_{3}\left(\omega_{1}, \omega_{2}\right)=\mathbf{j} \omega_{2}, H_{4}\left(\omega_{1}, \omega_{2}\right)=\mathbf{k} \omega_{1} \omega_{2}$. By [], we have $g_{1}\left(x_{1}, x_{2}\right)=f\left(x_{1}, x_{2}\right), g_{2}\left(x_{1}, x_{2}\right)=\frac{\partial f}{\partial x_{1}}\left(x_{1},-x_{2}\right), g_{3}\left(x_{1}, x_{2}\right)=\frac{\partial f}{\partial x_{2}}\left(x_{1}, x_{2}\right), g_{4}\left(x_{1}, x_{2}\right)=$ $-\frac{\partial^{2} f}{\partial x_{1} x_{2}}\left(x_{1},-x_{2}\right)$. Furthermore,

$$
\underline{H}=\left(\begin{array}{cccc}
1 & \mathbf{i} \omega_{1} & \mathbf{j} \omega_{2} & \mathbf{k} \omega_{1} \omega_{2} \\
1 & \mathbf{i} \omega_{1} & \mathbf{j}\left(\omega_{2}+c\right) & \mathbf{k} \omega_{1}\left(\omega_{2}+c\right) \\
1 & \mathbf{i}\left(\omega_{1}+c\right) & \mathbf{j} \omega_{2} & \mathbf{k}\left(\omega_{1}+c\right) \omega_{2} \\
1 & \mathbf{i}\left(\omega_{1}+c\right) & \mathbf{j}\left(\omega_{2}+c\right) & \mathbf{k}\left(\omega_{1}+c\right)\left(\omega_{2}+c\right)
\end{array}\right)=A_{1}+A_{2} \mathbf{j}
$$

where $A_{1}=\left(\begin{array}{cccc}1 & \mathbf{i} \omega_{1} & 0 & 0 \\ 1 & \mathbf{i} \omega_{1} & 0 & 0 \\ 1 & \mathbf{i}\left(\omega_{1}+c\right) & 0 & 0 \\ 1 & \mathbf{i}\left(\omega_{1}+c\right) & 0 & 0\end{array}\right)$ and $A_{2}=\left(\begin{array}{cccc}0 & 0 & \omega_{2} & \mathbf{i} \omega_{1} \omega_{2} \\ 0 & 0 & \left(\omega_{2}+c\right) & \mathbf{i} \omega_{1}\left(\omega_{2}+c\right) \\ 0 & 0 & \omega_{2} & \mathbf{i}\left(\omega_{1}+c\right) \omega_{2} \\ 0 & 0 & \left(\omega_{2}+c\right) & \mathbf{i}\left(\omega_{1}+c\right)\left(\omega_{2}+c\right)\end{array}\right)$. The complex adjoint matrix [20] of $\underline{H}$ denoted by $C(\underline{H})$ is defined as

$$
C(\underline{H})=\left(\begin{array}{cc}
A_{1} & A_{2} \\
-\overline{A_{2}} & \overline{A_{1}}
\end{array}\right) .
$$

Zhang [20] showed that $\underline{H}$ is invertible if and only if $C(\underline{H})$ is invertible. Moreover, $C\left(\underline{H}^{-1}\right)=[C(\underline{H})]^{-1}$ if $\underline{H}^{-1}$ exsits. Since $\left.\mid C(\underline{H})\right] \mid=c^{8} \neq 0$ for every $\left(\omega_{1}, \omega_{2}\right) \in I$. Therefore $\underline{H}$ is invertible for every $\left(\omega_{1}, \omega_{2}\right) \in I$. In fact, $[C(\underline{H})]^{-1}=\left(U_{1}, U_{2}\right)$ where

$$
U_{1}=\left(\begin{array}{cccc}
\frac{\left(c+\omega_{2}\right)\left(c+\omega_{1}\right)}{c^{2}} & -\frac{\omega_{2}\left(c+\omega_{1}\right)}{c^{2}} & -\frac{\left(c+\omega_{2}\right) \omega_{1}}{c^{2}} & \frac{\omega_{1} \omega_{2}}{c^{2}} \\
\frac{\mathbf{i}\left(c+\omega_{2}\right)}{c^{2}} & -\frac{\mathbf{i} \omega_{2}}{c^{2}} & -\frac{\mathbf{i}\left(c+\omega_{2}\right)}{c^{2}} & \frac{\mathbf{i} \omega_{2}}{c^{2}} \\
0 & 0 & 0 & 0 \\
0 & 0 & 0 & 0 \\
0 & 0 & 0 & 0 \\
0 & 0 & 0 & 0 \\
-\frac{c+\omega_{1}}{c^{2}} & \frac{c+\omega_{1}}{c^{2}} & \frac{\omega_{1}}{c^{2}} & -\frac{\omega_{1}}{c^{2}} \\
-\frac{\mathbf{i}}{c^{2}} & \frac{\mathbf{i}}{c^{2}} & \frac{\mathbf{i}}{c^{2}} & -\frac{\mathbf{i}}{c^{2}}
\end{array}\right)
$$


and

$$
U_{2}=\left(\begin{array}{cccc}
0 & 0 & 0 & 0 \\
0 & 0 & 0 & 0 \\
\frac{c+\omega_{1}}{c^{2}} & -\frac{c+\omega_{1}}{c^{2}} & -\frac{\omega_{1}}{c^{2}} & \frac{\omega_{1}}{c^{2}} \\
-\frac{\mathbf{i}}{c^{2}} & \frac{\mathbf{i}}{c^{2}} & \frac{\mathbf{i}}{c^{2}} & -\frac{\mathbf{i}}{c^{2}} \\
\frac{\left(c+\omega_{2}\right)\left(c+\omega_{1}\right)}{c^{2}} & -\frac{\omega_{2}\left(c+\omega_{1}\right)}{c^{2}} & -\frac{\left(c+\omega_{2}\right) \omega_{1}}{c^{2}} & \frac{\omega_{2} \omega_{1}}{c^{2}} \\
-\frac{\mathbf{i}\left(c+\omega_{2}\right)}{c^{2}} & \frac{\mathbf{i} \omega_{2}}{c^{2}} & \frac{\mathbf{i}\left(c+\omega_{2}\right)}{c^{2}} & -\frac{\mathbf{i} v}{c^{2}} \\
0 & 0 & 0 & 0 \\
0 & 0 & 0 & 0
\end{array}\right) .
$$

Thus

$$
\underline{H}^{-1}=\left(\begin{array}{cccc}
\frac{\left(c+\omega_{2}\right)\left(c+\omega_{1}\right)}{c^{2}} & -\frac{\omega_{2}\left(c+\omega_{1}\right)}{c^{2}} & -\frac{\left(c+\omega_{2}\right) \omega_{1}}{c^{2}} & \frac{\omega_{2} \omega_{1}}{c^{2}} \\
\frac{\mathbf{i}\left(c+\omega_{2}\right)}{c^{2}} & -\frac{\mathbf{i} \omega_{2}}{c^{2}} & -\frac{\mathbf{i}\left(c+\omega_{2}\right)}{c^{2}} & \frac{1 \mathbf{i} \omega_{2}}{c^{2}} \\
\frac{\mathbf{j}\left(c+\omega_{1}\right)}{c^{2}} & -\frac{\mathbf{j}\left(c+\omega_{1}\right)}{c^{2}} & -\frac{\mathbf{j} \omega_{1}}{c^{2}} & \frac{-\mathbf{j} \omega_{1}}{c^{2}} \\
-\frac{\mathbf{k}}{c^{2}} & \frac{\mathbf{k}}{c^{2}} & \frac{\mathbf{k}}{c^{2}} & -\frac{\mathbf{k}}{c^{2}}
\end{array}\right)
$$

and hence

$$
\begin{aligned}
& \frac{1}{2 \pi} Y_{1}\left(\omega_{1}, \omega_{2}\right) \\
= & \sigma^{-4}\left(c+\omega_{2}\right)\left(c+\omega_{1}\right) \chi_{I_{11}}\left(\omega_{1}, \omega_{2}\right)-\sigma^{-4}\left(\omega_{2}-c\right)\left(c+\omega_{1}\right) \chi_{I_{12}}\left(\omega_{1}, \omega_{2}\right) \\
- & \sigma^{-4}\left(c+\omega_{2}\right)\left(\omega_{1}-c\right) \chi_{I_{21}}\left(\omega_{1}, \omega_{2}\right)+\sigma^{-4}\left(\omega_{2}-c\right)\left(\omega_{1}-c\right) \chi_{I_{22}}\left(\omega_{1}, \omega_{2}\right) .
\end{aligned}
$$

By direct computation,

$$
y_{1}\left(x_{1} \ominus n_{1} T, x_{2} \ominus n_{2} T\right)=\frac{16 \sin ^{2}\left(\frac{\sigma}{2} x_{1}-n_{1} \pi\right) \sin ^{2}\left(\frac{\sigma}{2} x_{2}-n_{2} \pi\right)}{\sigma^{4}\left(x_{1}-n_{1} T\right)^{2}\left(x_{2}-n_{2} T\right)^{2}} .
$$

Similarly, we have

$$
\begin{aligned}
& y_{2}\left(x_{1} \ominus n_{1} T, x_{2} \ominus n_{2} T\right)=\frac{16 \sin ^{2}\left(\frac{\sigma}{2} x_{1}-n_{1} \pi\right) \sin ^{2}\left(\frac{\sigma}{2} x_{2}+n_{2} \pi\right)}{\sigma^{4}\left(x_{1}-n_{1} T\right)\left(x_{2}+n_{2} T\right)^{2}}, \\
& y_{3}\left(x_{1} \ominus n_{1} T, x_{2} \ominus n_{2} T\right)=\frac{16 \sin ^{2}\left(\frac{\sigma}{2} x_{1}-n_{1} \pi\right) \sin ^{2}\left(\frac{\sigma}{2} x_{2}-n_{2} \pi\right)}{\sigma^{4}\left(x_{1}-n_{1} T\right)^{2}\left(x_{2}-n_{2} T\right)}, \\
& y_{4}\left(x_{1} \ominus n_{1} T, x_{2} \ominus n_{2} T\right)=-\frac{16 \sin ^{2}\left(\frac{\sigma}{2} x_{1}-n_{1} \pi\right) \sin ^{2}\left(\frac{\sigma}{2} x_{2}+n_{2} \pi\right)}{\sigma^{4}\left(x_{1}-n_{1} T\right)\left(x_{2}+n_{2} T\right)} .
\end{aligned}
$$

\section{Sampling theorem for Quaternion linear canonical transform}

The right-sided quaternion linear canonical transform (QLCT) which is generalization of linear canonical transform (LCT) to quaternion algebra, was firstly studied in [21]. In this section, we investigate the sampling theory associated with QLCT. The right-sided QLCT of a signal $f \in L^{1}\left(\mathbb{R}^{2}, \mathbb{H}\right)$ with real matrix parameter $A_{i}=\left(\begin{array}{ll}a_{i} & b_{i} \\ c_{i} & d_{i}\end{array}\right) \in \mathbb{R}^{2 \times 2}$ such 
that $\operatorname{det}\left(A_{i}\right)=1$ for $i=1,2$ is defined by [21]

$$
(\mathcal{L} f)\left(\omega_{1}, \omega_{2}\right):=\int_{\mathbb{R}^{2}} f\left(x_{1}, x_{2}\right) K_{A_{1}}^{\mathbf{i}}\left(x_{1}, \omega_{1}\right) K_{A_{2}}^{\mathbf{j}}\left(x_{2}, \omega_{2}\right) d x_{1} d x_{2}
$$

where

$$
K_{A_{1}}^{\mathbf{i}}\left(x_{1}, \omega_{1}\right):=\frac{1}{\sqrt{\mathbf{i} 2 \pi b_{1}}} \mathbf{e}^{\mathbf{i}\left(\frac{a_{1}}{2 b_{1}} x_{1}^{2}-\frac{1}{b_{1}} x_{1} \omega_{1}+\frac{d_{1}}{2 b_{1}} w_{1}^{2}\right)}, \quad \text { for } \quad b_{1} \neq 0
$$

and

$$
K_{A_{2}}^{\mathbf{j}}\left(x_{2}, \omega_{2}\right):=\frac{1}{\sqrt{\mathbf{j} 2 \pi b_{2}}} \mathbf{e}^{\mathbf{j}\left(\frac{a_{2}}{2 b_{2}} x_{2}^{2}-\frac{1}{b_{2}} x_{2} \omega_{2}+\frac{d_{2}}{2 b_{2}} w_{2}^{2}\right)}, \quad \text { for } \quad b_{2} \neq 0
$$

Here, $\frac{1}{\sqrt{\mu 2 \pi b}}$ represents $|2 \pi b|^{\frac{-1}{2}} \mathbf{e}^{\mu \frac{\operatorname{sgn} b-2}{4} \pi}$ for any pure imaginary unit quaternion $\boldsymbol{\mu}$ and nonzero real number $b$.

If $\mathcal{L} f$ is also in $L^{1}\left(\mathbb{R}^{2}, \mathbb{H}\right)$, the the inversion QLCT formula [17] holds, that is

$$
f\left(x_{1}, x_{2}\right)=\left(\mathcal{L}^{-1} \mathcal{L} f\right)\left(x_{1}, x_{2}\right):=\int_{\mathbb{R}^{2}}(\mathcal{L} f)\left(\omega_{1}, \omega_{2}\right) K_{A_{2}^{-1}}^{\mathbf{j}}\left(\omega_{2}, x_{2}\right) K_{A_{1}^{-1}}^{\mathbf{i}}\left(\omega_{1}, x_{1}\right) d \omega_{1} d \omega_{2},
$$

for almost every $\left(x_{1}, x_{2}\right) \in \mathbb{R}^{2}$. By Plancherel theorem [17], the QLCT can be extended to $L^{2}\left(\mathbb{R}^{2}, \mathbb{H}\right)$. As an operator on $L^{2}\left(\mathbb{R}^{2}, \mathbb{H}\right)$, the QLCT $\Phi$ is a bijection and the Parseval's identity $\|\Phi f\|_{2}=\|f\|_{2}$ holds. Since $\Phi\left(\Phi^{-1}\right)$ coincides with $\mathcal{L}\left(\mathcal{L}^{-1}\right)$ in $L^{1}\left(\mathbb{R}^{2}, \mathbb{H}\right) \cap$ $L^{2}\left(\mathbb{R}^{2}, \mathbb{H}\right)$. So we use letter $F_{\mathbf{A}}$ to denote the QLCT of $f \in L^{1}\left(\mathbb{R}^{2}, \mathbb{H}\right) \cup L^{2}\left(\mathbb{R}^{2}, \mathbb{H}\right)$.

In the classical case, the LCT is just a variation of the standard Fourier transform, some of its properties can be deduced from those of the Fourier transform by a change of variable. Moreover, the proofs of many sampling formulae associated with the LCT are somewhat based on those of the Fourier transform. In the quaternionic case, however, $\mathcal{L}$ can not directly establish relation with $\mathcal{F}$ as mentioned in [17]. So it is hard to derive GSE associated with the QLCT from existing results of the QFT. On the other hand, Lemma 4.2 is based on periodicity of kernel $\mathbf{e}^{\mathrm{j} \omega_{2} x_{2}} \mathbf{e}^{\mathrm{i} \omega_{1} x_{1}}$, but due to the quadratic term of kernel (6.1) and (6.2) in the QLCT, periodicity of kernel no longer possess. When $M>1$, (4.5) of Theorem 4.4 is also called multichannel sampling expansion. In the following, we give a single-channel sampling expansion associated with the QLCT, that is we only focus on the case of $M=1$.

Firstly, we introduce the generalized translation related to the QLCT:

$$
\begin{aligned}
& f\left(x_{1} \boxminus y_{1}, x_{2} \boxminus y_{2}\right) \\
:= & \int_{\mathbb{R}^{2}} \frac{K_{A_{2}^{-1}}^{\mathbf{j}}\left(\omega_{2}, y_{2}\right) K_{A_{1}^{-1}}^{\mathbf{i}}\left(\omega_{1}, y_{1}\right)}{F_{\mathbf{A}}}\left(\omega_{2}, \omega_{2}\right) K_{A_{2}^{-1}}^{\mathbf{j}}\left(\omega_{2}, x_{2}\right) K_{A_{1}^{-1}}^{\mathbf{i}}\left(\omega_{1}, x_{1}\right) d \omega_{1} d \omega_{2}
\end{aligned}
$$

provided taht the right-hand side integral is well defined. Then we have the following theorem.

Theorem 6.1 Suppose that $f$ is $\sigma$-bandlimited in QLCT sense, that is

$$
f\left(x_{1}, x_{2}\right)=\int_{I} F_{\mathbf{A}}\left(\omega_{2}, \omega_{2}\right) K_{A_{2}^{-1}}^{\mathbf{j}}\left(\omega_{2}, x_{2}\right) K_{A_{1}^{-1}}^{\mathbf{i}}\left(\omega_{1}, x_{1}\right) d \omega_{1} d \omega_{2}
$$


where $F_{\mathbf{A}} \in L^{2}(I, \mathbb{H})$ and $I=[-\sigma, \sigma]^{2}$. Let

$$
g\left(x_{1}, x_{2}\right)=\int_{I} F_{\mathbf{A}}\left(\omega_{2}, \omega_{2}\right) H\left(\omega_{1}, \omega_{2}\right) K_{A_{2}^{-1}}^{\mathbf{j}}\left(\omega_{2}, x_{2}\right) K_{A_{1}^{-1}}^{\mathbf{i}}\left(\omega_{1}, x_{1}\right) d \omega_{1} d \omega_{2}
$$

where $H\left(\omega_{1}, \omega_{2}\right), H\left(\omega_{1}, \omega_{2}\right)^{-1} \in L^{2}(I, \mathbb{H})$. Let

$$
Y_{\mathbf{A}}\left(\omega_{1}, \omega_{2}\right)=T^{2}\left|b_{1} b_{2}\right| H\left(\omega_{1}, \omega_{2}\right)^{-1} \chi_{I}\left(\omega_{1}, \omega_{2}\right) .
$$

Then $f$ can be reconstructed from samples $g\left(n_{1} b_{1} T, n_{2} b_{2} T\right)$ :

$$
f\left(x_{1}, x_{2}\right)=\sum_{n_{1}, n_{2}} g\left(n_{1} b_{1} T, n_{2} b_{2} T\right) y\left(x_{1} \boxminus n_{1} b_{1} T, x_{2} \boxminus n_{2} b_{2} T\right)
$$

where $y$ is the inverse $Q L C T$ of $Y_{\mathbf{A}}$ and $T=\frac{\pi}{\sigma}$.

Proof. We note that $\varphi_{n_{1} n_{2}}\left(\omega_{1}, \omega_{2}\right):=T\left|b_{1} b_{2}\right|^{\frac{1}{2}} \overline{K_{A_{2}^{-1}}^{\mathbf{j}}\left(\omega_{2}, n_{2} b_{2} T\right) K_{A_{1}^{-1}}^{\mathbf{i}}\left(\omega_{1}, n_{1} b_{1} T\right)}$ is an orthonormal basis of $L^{2}(I, \mathbb{H})$. Therefore by invoking $(2.1)$ we have

$$
\begin{aligned}
f\left(x_{1}, x_{2}\right)= & \int_{I} F_{\mathbf{A}}\left(\omega_{2}, \omega_{2}\right) K_{A_{2}^{-1}}^{\mathbf{j}}\left(\omega_{2}, x_{2}\right) K_{A_{1}^{-1}}^{\mathbf{i}}\left(\omega_{1}, x_{1}\right) d \omega_{1} d \omega_{2} \\
= & \int_{I} F_{\mathbf{A}}\left(\omega_{2}, \omega_{2}\right) H\left(\omega_{1}, \omega_{2}\right) H\left(\omega_{1}, \omega_{2}\right)^{-1} K_{A_{2}^{-1}}^{\mathbf{j}}\left(\omega_{2}, x_{2}\right) K_{A_{1}^{-1}}^{\mathbf{i}}\left(\omega_{1}, x_{1}\right) d \omega_{1} d \omega_{2} \\
= & \sum_{n_{1}, n_{2}}\left(\int_{I} F_{\mathbf{A}}\left(\omega_{2}, \omega_{2}\right) H\left(\omega_{1}, \omega_{2}\right) \overline{\varphi_{n_{1} n_{2}}\left(\omega_{1}, \omega_{2}\right)} d \omega_{1} d \omega_{2}\right) \\
& \left(\int_{I} \varphi_{n_{1} n_{2}}\left(\omega_{1}, \omega_{2}\right) H\left(\omega_{1}, \omega_{2}\right)^{-1} K_{A_{2}^{-1}}^{\mathbf{j}}\left(\omega_{2}, x_{2}\right) K_{A_{1}^{-1}}^{\mathbf{i}}\left(\omega_{1}, x_{1}\right) d \omega_{1} d \omega_{2}\right) \\
= & \sum_{n_{1}, n_{2}} g\left(n_{1} b_{1} T, n_{2} b_{2} T\right) y\left(x_{1} \boxminus n_{1} b_{1} T, x_{2} \boxminus n_{2} b_{2} T\right)
\end{aligned}
$$

which completes the proof.

Now we give an example for this Theorem. Suppose that $f$ is $\sigma$-bandlimited in QLCT sense and let $H\left(\omega_{1}, \omega_{2}\right)=1$, then $g\left(x_{1}, x_{2}\right)=f\left(x_{1}, x_{2}\right)$. Therefore

$$
f\left(x_{1}, x_{2}\right)=\sum_{n_{1}, n_{2}} f\left(n_{1} b_{1} T, n_{2} b_{2} T\right) y\left(x_{1} \boxminus n_{1} b_{1} T, x_{2} \boxminus n_{2} b_{2} T\right)
$$

By (6.3) we obtain $y\left(x_{1} \boxminus n_{1} b_{1} T, x_{2} \boxminus n_{2} b_{2} T\right)=\varrho_{1}+\varrho_{2} \varrho_{3}$ where $\varrho_{1}, \varrho_{2}, \varrho_{3}$, respectively, are

$$
\begin{aligned}
& \frac{T^{2} b_{1}\left|b_{2}\right|}{\pi^{2}} \cos \left(\frac{a_{2} b_{2} n_{2}^{2} T^{2}}{2}-\frac{a_{2} x_{2}^{2}}{2 b_{2}}\right) \frac{\sin \left(n_{1} \pi-\frac{\pi x_{1}^{2}}{b_{1} T}\right) \sin \left(n_{2} \pi-\frac{\pi x_{2}^{2}}{b_{2} T}\right)}{\left(n_{1} b_{1} T-x_{1}\right)\left(n_{2} b_{2} T-x_{2}\right)} \mathbf{e}^{\mathrm{i}\left(\frac{a_{1} b_{1} n_{1}^{2} T^{2}}{2}-\frac{a_{1} x_{1}^{2}}{2 b_{1}}\right)}, \\
& \operatorname{erf}\left(\frac{\left|b_{1} b_{2}\right|^{\frac{-1}{2}}}{2 T}\left(2 d_{1} \pi-b_{1} n_{1} T^{2}-x_{1} T\right)\right)+\operatorname{erf}\left(\frac{\left|b_{1} b_{2}\right|^{\frac{-1}{2}}}{2 T}\left(2 d_{1} \pi+b_{1} n_{1} T^{2}+x_{1} T\right)\right)
\end{aligned}
$$

and

$$
\left(\frac{\left|b_{1}\right|}{\pi}\right)^{\frac{3}{2}} \frac{T^{2}\left|d_{1}\right|^{\frac{-1}{2}}}{4} \sin \left(\frac{a_{2} b_{2} n_{2}^{2} T^{2}}{2}-\frac{a_{2} x_{2}^{2}}{2 b_{2}}\right) \frac{\sin \left(n_{2} \pi-\frac{\pi x_{2}^{2}}{b_{2} T}\right)}{n_{2} b_{2} T-x_{2}} \mathbf{e}^{\mathbf{i}\left(\frac{a_{1} b_{1} n_{1}^{2} T^{2}}{2}+\frac{a_{1} x_{1}^{2}}{2 b_{1}}-\frac{\left(b_{1} n_{1} T+x_{1}\right)^{2}}{4 b_{1} d_{1}}\right)} \mathbf{j} .
$$




\section{Conclusion}

In this paper, we introduced the GSE associated with QFT. The GSE formula illustrates how a bandlimited quaternion valued signal can be recovered from the samples of system output signals. This has been realized by taking advantage of generalized translation and convolution. Moreover, we have further discussed the sampling formula for $\sigma$-bandlimited quaternion valued signal in quaternion linear canonical transform sense.

\section{Acknowledgements}

The authors acknowledge financial support from the National Natural Science Foundation of China under Grant (No. 11401606), University of Macau (No. MYRG2015-00058-L2FST and No. MYRG099(Y1-L2)-FST13-KKI) and the Macao Science and Technology Development Fund (No. FDCT/094/2011/A and No. FDCT/099/2012/A3).

\section{References}

[1] A. Papoulis, "Generalized sampling expansion," IEEE Trans. Circuits Syst., vol. 24, no. 11, pp. 652-654, 1977.

[2] R. Hoskins and J. de Sousa Pinto, "Generalized sampling expansions in the sense of papoulis," SIAM J. Appl. Math., vol. 44, no. 3, pp. 611-617, 1984.

[3] K. F. Cheung, "A multidimensional extension of papoulis generalized sampling expansion with the application in minimum density sampling," in Advanced Topics in Shannon Sampling and Interpolation Theory, pp. 85-119, Springer, 1993.

[4] D. Wei, Q. Ran, and Y. Li, "Generalized sampling expansion for bandlimited signals associated with the fractional Fourier transform," IEEE Signal Process. Lett., vol. 17, no. 6, pp. 595-598, 2010.

[5] K. Shoemake, "Animating rotation with quaternion curves," in SIGGRAPH Comput. Graph., vol. 19, pp. 245-254, ACM, 1985.

[6] A. J. Hanson and H. Ma, "Quaternion frame approach to streamline visualization," IEEE Trans. Vis. Comput. Graphics, vol. 1, no. 2, pp. 164-174, 1995.

[7] X. Yun and E. R. Bachmann, "Design, implementation, and experimental results of a quaternion-based kalman filter for human body motion tracking," IEEE Trans. Robot., vol. 22, no. 6, pp. 1216-1227, 2006.

[8] S. J. Sangwine, "Fourier transforms of colour images using quaternion or hypercomplex, numbers," Electron. Lett., vol. 32, no. 21, pp. 1979-1980, 1996. 
[9] T. Bülow, Hypercomplex Spectral Signal Representations for The Processing and Analysis of Images. Universität Kiel. Institut für Informatik und Praktische Mathematik, 1999.

[10] N. L. Bihan and S. J. Sangwine, "Quaternion principal component analysis of color images," in Image Processing, 2003. ICIP 2003. Proceedings. 2003 International Conference on, vol. 1, pp. I-809, IEEE, 2003.

[11] N. Le Bihan and J. Mars, "Singular value decomposition of quaternion matrices: a new tool for vector-sensor signal processing," Signal Process., vol. 84, no. 7, pp. 11771199, 2004.

[12] T. Ell, S. J. Sangwine, et al., "Hypercomplex Fourier transforms of color images," IEEE Trans. Image Process., vol. 16, no. 1, pp. 22-35, 2007.

[13] F. Brackx, R. Delanghe, and F. Sommen, Clifford Analysis, vol. 76. Pitman Books Limited, 1982.

[14] R. Ghiloni, V. Moretti, and A. Perotti, "Continuous slice functional calculus in quaternionic Hilbert spaces," Rev. Math. Phys., vol. 25, no. 04, p. 1350006, 2013.

[15] T. A. Ell, "Quaternion-Fourier transforms for analysis of two-dimensional linear time-invariant partial differential systems," in Decision and Control, 1993., Proceedings of the 32nd IEEE Conference on, pp. 1830-1841, IEEE, 1993.

[16] T. A. Ell, "Quaternion Fourier transform: Re-tooling image and signal processing analysis," in Quaternion and Clifford Fourier Transforms and Wavelets, pp. 3-14, Springer, 2013.

[17] D. Cheng and K. I. Kou, "Properties of quaternion fourier transforms," Preprint.

[18] E. M. Hitzer, "Quaternion Fourier transform on quaternion fields and generalizations," Adv. Appl. Clifford Alg., vol. 17, no. 3, pp. 497-517, 2007.

[19] R. J. Marks II, Advanced topics in Shannon sampling and interpolation theory. Springer Science \& Business Media, 2012.

[20] F. Zhang, "Quaternions and matrices of quaternions," Linear Algebra Appl., vol. 251, pp. 21-57, 1997.

[21] K. I. Kou, J.-Y. Ou, and J. Morais, "On uncertainty principle for quaternionic linear canonical transform," in Abstract and Applied Analysis, vol. 2013, Hindawi Publishing Corporation, 2013. 